\title{
Análise comparativa inicial de critérios oncológicos de 120 pacientes submetidos a cirurgias colorretais por via laparotômica (60 pacientes) e por via videolaparoscópica (60 pacientes) para câncer colorretal no Programa de Pós-graduação sensu lato pelo Grupo de Coloproctologia de Belo Horizonte
}

\author{
Initial analysis of oncologic criteria of 120 patients undergone colorectal \\ laparotomic (60 patients) and videolaparoscopic surgeries (60 patients) for \\ colorectal cancer in a Postgraduate Program (residency) by the Group of \\ Coloproctology of Belo Horizonte
}

\begin{abstract}
JOSÉ ROBERTO MONTEIRO CONSTANTINO ${ }^{1}$, PETERSON MARTINS NEVES ${ }^{1}$, ANTONIO CARLOS BARROS LIMA JUNIOR ${ }^{2}$, FABIO GONTIJO RODRIGUES ${ }^{2}$, GUILHERME DE ALMEIDA SANTOS ${ }^{2}$, CAROLINE PINTO COUTINHO $^{2}$, FLAVIA FONTES FARIA ${ }^{2}$, RODRIGO GUIMARÃES OLIVEIRA ${ }^{2}$, ESTEVAN GUILLERMO VIGIL VERASTEGUI SILVA ${ }^{3}$, ÁUREA CÁSSIA GUALBETO BRAGA ${ }^{1}$, RENATA MAGALI RIBEIRO SILLUZIO FERREIRA $^{1}$, ISABELLA MENDONÇA ALVARENGA ${ }^{1}$, DAVID DE LANNA ${ }^{1}$, RICARDO GUIMARÃES TEIXEIRA ${ }^{1}$, HERALDO NEVES VALLE JUNIOR ${ }^{1}$, SINARA MÔNICA OLIVEIRA LEITE ${ }^{1}$, LUCIANA MARIA PYRAMO COSTA ${ }^{1}$, ILSON GERALDO DA SILVA ${ }^{4}$, GERALDO MAGELA GOMES DA CRUZ
\end{abstract}

\begin{abstract}
${ }^{1}$ Assistentes efetivos do Grupo de Coloproctologia da Santa Casa de Belo Horizonte e Faculdade de Ciências Médicas de Minas Gerais - Minas Gerais (MG), Brasil. ${ }^{2}$ Residentes do Grupo de Coloproctologia da Santa Casa de Belo Horizonte e Faculdade de Ciências Médicas de Minas Gerais - Minas Gerais (MG), Brasil. ${ }^{3}$ Estagiário voluntário do Grupo de Coloproctologia da Santa Casa de Belo Horizonte e Faculdade de Ciências Médicas de Minas Gerais - Minas Gerais $(M G)$, Brasil. ${ }^{4}$ Chefe interino do Serviço de Coloproctologia de Homens da Santa Casa de Belo Horizonte - Minas Gerais $(M G)$, Brasil. ${ }^{5}$ Chefe do Serviço de Coloproctologia de Mulheres e do Ambulatório de Coloproctologia da Santa Casa de Belo Horizonte - Minas Gerais (MG), Brasil; Coordenador do Grupo de Coloproctologia da Santa Casa de Belo Horizonte e Faculdade de Ciências Médicas de Minas Gerais - Minas Gerais (MG), Brasil.
\end{abstract}

CONSTANTINO JRM, NEVES PM, JUNIOR ACBL, RODRIGUES FG, SANTOS GDA, COUTINHO CP, FARIA FF, OLIVEIRA RG, SILVA EGVV, BRAGA ÁCG, FERREIRA RMRS, ALVARENGA IM, LANNA DD, TEIXEIRA RG, JUNIOR HNV, LEITE SMO, COSTA LMP, SILVA IGD, CRUZ GMGD. Análise comparativa inicial de critérios oncológicos de 120 pacientes submetidos a cirurgias colorretais por via laparotômica (60 pacientes) e por via videolaparoscópica (60 pacientes) para câncer colorretal no Programa de Pós-graduação sensu lato pelo Grupo de Coloproctologia de Belo Horizonte. Rev bras Coloproct, 2011;31(2): 184-196.

RESUMO: O objetivo do trabalho foi proceder a uma revisão criteriosa de uma casuística de 120 pacientes portadores de câncer colorretal operados por via videolaparoscópica (grupo-Video, 60 pacientes) e por via laparotômica (grupo-Lap, com 60 pacientes), no decurso de 12 meses (maio de 2009 a maio de 2010). A média etária foi de 58 anos, sendo 58,8 anos no grupo-Lap e 57,3 no grupo-Video, e a maioria era do sexo feminino em ambos os grupos (grupo-Lap - 55,0\% e grupo-Video - 61,7\%, com média de 58,3 anos). Todos os pacientes de ambos os grupos foram submetidos à colonoscopia e biópsia, com diagnóstico histopatológico de adenocarcinoma. Na distribuição dos tumores pelo intestino grosso no grupo-Lap, 43 se localizavam no reto e sigmoide (71,7\%) contra 45 no grupo-Video $(\mathbf{7 5 , 0 \%}$ ), mas com diferenças entre reto baixo (grupo-Lap, 13,3\%; grupo-Video, 16,7\%) e alto (grupo-Lap, 30,0\%; grupo-Video, $16,7 \%$ ), sigmoide e retossigmoide (grupo-Lap, 28,4\%; grupo-Video, 41,6\%). As cirurgias mais realizadas foram as retossigmoidec-

Trabalho realizado pelo Grupo de Coloproctologia da Santa Casa de Belo Horizonte e Faculdade de Ciências Médicas de Minas Gerais - Minas Gerais $(M G)$, Brasil. 
tomias abdominais altas (grupo-Lap, 27 casos, 45,0\%; grupo-Video, 33 casos, 55,0\%), seguidas pelas hemicolectomias direitas com anastomose íleo-transverso (grupo-Lap, 16 casos, 26,6\%; e grupo-Video, 13 casos, 21,7\%). As extensões das peças cirúrgicas foram maiores no grupo-Lap (média de $46,1 \mathrm{~cm}$ contra $30,0 \mathrm{~cm}$ no grupo-Video) em decorrência de maior número de cirurgias que resultaram peças de grandes dimensões. Quando se comparam as mesmas técnicas cirúrgicas, a diferença não persiste, como nos casos das retossigmoidectomias (grupo-Lap com 32 casos, média de $28,2 \mathrm{~cm}$; grupo-Video com 39 casos, com média de 26,6 cm). No tocante à gradação TNM dos tumores, a gradação T3N0M0 foi a mais comum: grupo-Lap com 30 casos (50,0\%) e grupo-Video com 35 casos $(58,4 \%)$. No tocante à contagem de linfonodos nas peças cirúrgicas, nenhuma diferença foi notada: total de 810 linfonodos no grupoLap, com média de 13,5 linfonodos por peça, e total de 862 linfonodos no grupo-Video, com média de 14,3 linfonodos por peça. Não houve diferença também no tocante à contagem de linfonodos nas peças cirúrgicas, mais comuns entre 11 e 15: 34 casos no grupoLap (56,7\%) e 38 no grupo-Video (63,3\%). Assim, nenhuma diferença foi notada entre os dois grupos (grupo-Lap e grupo-Video) no tocante aos critérios oncológicos cirúrgicos.

Palavras-chave: câncer retal; laparoscopia; cirurgia colorretal.

\section{INTRODUÇÃO}

Os procedimentos cirúrgicos por videolaparoscopia são realizados, atualmente, como rotina em muitos serviços tal a experiência e a segurança acumuladas ao longo dos últimos 20 anos, não havendo dúvidas de que acarretam uma melhor resposta ao trauma, tanto sob o ponto de vista doloroso quanto inflamatório, endócrino e metabólico, quando comparados aos procedimentos laparotômicos ${ }^{1}$. Todavia, é técnica que se reveste de características próprias e extremamente peculiares, tanto no tocante ao treinamento dos cirurgiões quanto aos recursos alocados e aspectos relacionados à sua prática. Em decorrência disso, não é raro que vários especialistas de um determinado serviço pratiquem a técnica em vários hospitais, enquanto o próprio serviço não o pratique em sua própria sede pelos motivos mais variados, o que inclui a indisponibilidade dos recursos necessários. Assim, embora vários especialistas do Grupo de Coloproctologia da Santa Casa de Belo Horizonte e Faculdade de Ciências Médicas de Minas Gerais (GCP) se dedicassem à cirurgia videolaparoscópica em outros hospitais, somente no início de 2009 a técnica foi incorporada por todos os membros do Grupo e inserida na pós-graduação da especialidade na Santa Casa de Belo Horizonte.

Assim, dentro do Programa de Pós-graduação em Coloproctologia sensu lato, a partir do mês de maio de 2009, o GCP, após um período variado de aprendizagem e treinamento, iniciou a prática rotineira de cirurgia videolaparoscópica para abordagem das doenças colorretais, mesmo ainda em números muito inferiores que aos de cirurgias laparotômicas. Duran- te este período de 12 meses (maio de 2009 a maio de 2010), foram operados, pelo GCP, por essa técnica, 90 pacientes, o que equivale a cerca de oito cirurgias videolaparoscópicas por mês, que corresponde a pouco menos da metade das cirurgias laparotômicas no mesmo período. Provavelmente, essa proporção deverá cair nos anos vindouros certamente em decorrência da diminuição dos critérios de exclusão atualmente usados. Dentre esses 90 pacientes, 60 foram abordados por câncer colorretal.

Videolaparoscopia: O advento da cirurgia videolaparoscópica foi de suma importância para a cirurgia gastrointestinal nos últimos 20 anos. A primeira colecistectomia videolaparoscópica foi realizada em 1987; em pouco tempo obteve grande aceitação e tornou-se a cirurgia mais realizada no mundo. Aos poucos, essa técnica foi ganhando espaço em várias outras doenças abordadas cirurgicamente. Não surpreendentemente a utilização da técnica minimamente invasiva ganhou espaço na cirurgia colorretal ${ }^{2,3}$.

A partir da primeira colectomia por vídeo em 1991, a cirurgia videolaparoscópica passou a ser utilizada em várias doenças de natureza benigna, como pólipos, doença diverticular, doenças inflamatórias intestinais, prolapso retal e, posteriormente, em cânceres colorretais ${ }^{1,3}$.

Embora tenha sido aceita de forma relativamente rápida para abordagem de doenças benignas, seu uso na abordagem de doenças malignas foi polêmico e muito discutível. Somente aos poucos, com base em resultados convincentes, foi a videolaparoscopia aceita, com reservas, para o câncer colorretal. Ainda há reservas no tocante a essa técnica quando se trata do câncer retal. 
Dentre alguns benefícios da técnica, em comparação com a cirurgia convencional, estão a diminuição da dor pós-operatória, recuperação mais rápida com menor tempo de internação e/ou permanência hospitalar, e reinício da função intestinal ${ }^{1}$.

A técnica é complexa, exige aprendizado especial e destreza do cirurgião para a dissecção de planos intra-abdominais, identificação, ligadura, secção, anastomose de vasos sanguíneos e do cólon.

A melhoria do material e qualidade da resolução da imagem tem facilitado a realização de tal técnica, tornando-a cada vez mais aceita e usada.

À medida que a técnica era utilizada pelo mundo todo, os critérios de exclusão de pacientes foram reduzindo. As contraindicações das cirurgias colorretais por videolaparoscopia são as mesmas da cirurgia convencional, principalmente quando relacionadas com complicações cardiopulmonares. Pacientes obesos que antes eram contraindicados a laparoscopia podem hoje ser abordados por essa via, melhor opção aberta que a via convencional ${ }^{4}$.

Câncer retal: Os tumores malignos que acometem o cólon e o reto representam o segundo tipo de neoplasia mais prevalente no mundo, após o câncer de mama, com uma estimativa de 2,4 milhões de casos nos últimos cinco anos, ou seja, a cada ano estimam-se em 945 mil casos novos ${ }^{5}$.

Nos Estados Unidos da América (EUA), o câncer colorretal foi responsável por 285.040 óbitos no período de 1998 a 2002, ocupando o segundo lugar dentre os óbitos por neoplasia ${ }^{4}$. No Brasil, a mortalidade causada por câncer de cólon, reto e canal anal, em 2002, foi de 8.772 óbitos $^{6}$.

As estimativas para o ano de 2005 apontaram a neoplasia colorretal como a quarta mais frequente para ambos os sexos, sendo 12.410 casos novos em homens e 13.640 em mulheres. Esses valores correspondem a um risco estimado de 14 casos novos a cada 100 mil homens e de 15 para cada 100 mil mulheres. A maior incidência de casos ocorre na faixa etária entre 50 e 70 anos, com risco aumentado a partir dos 40 anos. Para esse tipo de neoplasia, a sobrevida média cumulativa em cinco anos é de $40-50 \%$, não sendo grande a diferença entre os países desenvolvidos e os em desenvolvimento ${ }^{5-7}$.

Programas de vigilância e proteção, assim como novas técnicas terapêuticas e normatização das técnicas cirúrgicas (e.g., excisão do mesorreto) contribuíram para a melhora do prognóstico do câncer de cólon e do reto. A cirurgia continua sendo a modalidade de tratamento primário de câncer colorretal.

Foi provado que a colectomia laparoscópica apresenta resultados semelhantes à cirurgia convencional.

As reoperações colorretais são consideradas um fator limitante ao acesso laparoscópico devido à fibrose com aderências e perda do plano anatômico ${ }^{4}$.

\section{OBJETIVO}

O objetivo do trabalho foi proceder a um levantamento minucioso de uma casuística de 120 pacientes portadores de câncer colorretal submetidos à abordagem cirúrgica por via laparotômica (grupo-Lap, com 60 pacientes) e por via videolaparoscópica (grupo-Video, com 60 pacientes), eletivamente, no decurso de 12 meses (maio de 2009 a maio de 2010).

\section{CASUÍSTICA - MATERIAIS E MÉTODOS}

Os 120 pacientes portadores de câncer colorretal objetos deste estudo foram extraídos de duas casuísticas - uma de 74 pacientes abordados por laparotomia por câncer colorretal (excluídos 14 pacientes operados em urgência e emergência, foram incluídos 60 ); e outra de 60 pacientes abordados, paralelamente, por videolaparoscopia por câncer colorretal - dentro do programa de pós-graduação em Coloproctologia sensu lato do Grupo de Coloproctologia da Santa Casa de Belo Horizonte e Faculdade de Ciências Médicas de Minas Gerais (GCP-SCBH-FCMMG), durante 12 meses (maio de 2009 a maio de 2010). Foi criado um protocolo de abordagem dos pacientes, contendo os seguintes dados, que foram minuciosamente seguidos pela equipe:

1. Gênero.

2. Idades e décadas etárias.

3. Localizações dos tumores no intestino grosso.

4. Cirurgias realizadas.

5. Extensão das peças cirúrgicas independentemente da cirurgia realizada (laudo anátomo-patológico).

6. Extensão das peças cirúrgicas dependendo da técnica usada (retossigmoidectomia abdominal 
com anastomose colorretal) (laudo anátomo-patológico).

7. Gradação dos tumores (TNM) (laudo anátomopatológico).

8. Contagem de linfonodos em peças cirúrgicas (laudo anátomo-patológico).

É importante ressaltar que foram adotados apenas dois critérios de exclusão: pacientes com laparotomias prévias e pacientes apresentando complicações emergenciais da doença de base abordada. Assim, foram inseridos no estudo 60 de 74 pacientes operados por via laparotômica (14 emergências) e todos os 60 operados por via videolaparoscópica.

O presente projeto foi aprovado pelo Comitê de Ética da Santa Casa de Belo Horizonte.

\section{RESULTADOS}

\section{Gêneros}

Houve mais mulheres (70 pacientes; 58,3\%) que homens (50 pacientes; $41,7 \%$ ) tanto no grupo-Lap (33 mulheres, 55,0\%; e 27 homens, 45,0\%) como no grupo-Video (37 mulheres, 61,7\%; e 23 homens, 38,3\%) (Tabela 1 e Figura 1).

\section{Idades e décadas etárias}

As décadas etárias com maior número de pacientes foram as quinta, sexta e sétima, assim distribuídas pelos grupos: grupo-Lap com 10 pacientes entre 41 e 50 anos $(16,7 \%), 17$ pacientes entre 51 e 60 anos $(28,2 \%)$ e 15 pacientes entre 61 e 70 anos (25\%), totalizando 42 pacientes $(69,9 \%)$; grupo-Video com 12 pacientes entre 41 e 50 anos $(20,0 \%), 19$ pacientes entre 51 e 60 anos $(31,7 \%)$ e 14 pacientes entre 61 e 70 anos $(23,3 \%)$, totalizando 45 pacientes $(75,0 \%)$; somandose os dois grupos, tem-se com 22 pacientes entre 41 e 50 anos (18,4\%), 36 pacientes entre 51 e 60 anos $(30,0 \%)$ e 29 pacientes entre 61 e 70 anos $(24,2 \%)$, totalizando 87 pacientes $(72,6 \%)$. A média das idades dos grupos foi a seguinte: 58,8 anos no grupo-Lap, 57,3 anos no grupo-Video e 58,0 anos nos dois grupos (Tabela 2 e Figura 2).

\section{Localizações dos tumores no intestino grosso}

A maioria dos 120 tumores se localizava no reto (46 casos; $38,3 \%$ ) e no sigmoide e retossigmoide (42 casos; $35 \%)$, totalizando 88 casos $(73,3 \%)$ em ambos
Tabela 1. Distribuição, por gêneros, de 120 pacientes portadores de câncer colorretal submetidos a cirurgias colorretais por laparotomia (60) e videolaparoscopia (60): houve 70 mulheres (58,3\%) e 50 homens $(41,7 \%)$, sendo que no grupo-Lap houve 33 mulheres $(55,0 \%)$ e 27 homens $(45,0 \%)$, e no grupo-Video houve 23 homens $(38,3 \%)$ e 37 mulheres $(61,7 \%)$.

\begin{tabular}{lcccccc}
\hline Gêneros & \multicolumn{2}{c}{ Grupo-Lap } & \multicolumn{2}{c}{$\begin{array}{c}\text { Grupo- } \\
\text { Video }\end{array}$} & \multicolumn{2}{c}{$\begin{array}{c}\text { Grupos } \\
\text { Lap+Video }\end{array}$} \\
\hline & $\mathbf{N}$ & $\mathbf{\%}$ & $\mathbf{N}$ & $\mathbf{\%}$ & $\mathbf{N}$ & $\mathbf{\%}$ \\
\hline Feminino & 33 & 55,0 & 37 & 61,7 & 70 & 58,3 \\
Masculino & 27 & 45,0 & 23 & 38,3 & 50 & 41,7 \\
Total & 60 & 100,0 & 60 & 100,0 & 120 & 100,0 \\
\hline
\end{tabular}

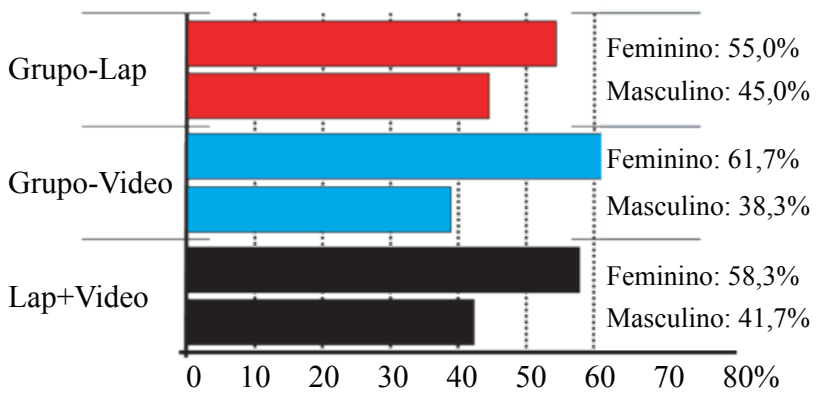

Figura 1. Distribuição, por gêneros, de 120 pacientes portadores de câncer colorretal submetidos a cirurgias colorretais por laparotomia (60) e videolaparoscopia (60): houve mais mulheres que homens, tanto entre os 120 pacientes quanto nos dois grupos (Lap e Video), como demonstrado nas colunas comparativas.

os grupos. Nos 60 pacientes do grupo-Lap, em 26 casos os tumores se localizavam no reto $(43,3 \%)$ e 17 se localizavam no retossigmoide e sigmoide $(28,4 \%)$, totalizando 43 (71,7\%); nos 60 pacientes do grupo-Video, 20 tumores se localizavam no reto $(33,4 \%)$ e 25 se localizavam no sigmoide e retossigmoide $(41,6 \%)$, totalizando $45(75,0 \%)$. Seguiu-se a localização cecal com 17 casos (14,2\%), sendo nove no grupo-Lap $(15,0 \%)$ e oito no grupo-Video $(13,3 \%)$ (Tabela 3 e Figura 3).

\section{Cirurgias realizadas}

Em decorrência das próprias localizações dos tumores no intestino grosso, a maioria das cirurgias foi representada pela retossigmoidectomia abdominal com anastomose colorretal alta, com o total de 60 casos $(50,0 \%)$, participando com 27 casos o grupo-Lap $(45,0 \%)$ e com 33 casos o grupo-Video 
Tabela 2. Distribuição, por décadas etárias, de 120 pacientes portadores de câncer colorretal submetidos a cirurgias colorretais por laparotomia (60) e videolaparoscopia (60): as médias etárias ficaram em 58,8 anos no grupo-Lap, 57,3 anos no grupo-Video e 58,0 anos nos 12 pacientes. As faixas prevalentes foram a de 41-70 anos para todos os grupos (grupo-Lap 69,9\%, grupo-Video 75,0\% e os dois grupos 72,6\%).

\begin{tabular}{lcccccc}
\hline $\begin{array}{l}\text { Idades (anos) } \\
\text { (Décadas) }\end{array}$ & $\begin{array}{c}\text { Grupo-Lap } \\
\text { Média: 58,8 anos }\end{array}$ & $\begin{array}{c}\text { Grupo-Video } \\
\text { Média: 57,3 anos }\end{array}$ & \multicolumn{2}{c}{$\begin{array}{c}\text { Lap+Video } \\
\text { Média: 58,0 anos }\end{array}$} \\
\hline & $\mathbf{N}$ & $\mathbf{\%}$ & $\mathbf{N}$ & $\mathbf{\%}$ & $\mathbf{N}$ & $\mathbf{\%}$ \\
\hline$<20$ & 00 & 0 & 00 & 0 & 00 & 0 \\
$21-30$ & 01 & 1,7 & 00 & 0 & 01 & 0,8 \\
$31-40$ & 04 & 6,7 & 05 & 8,3 & 09 & 7,5 \\
$41-50$ & 10 & 16,7 & 12 & 20,0 & 22 & 18,4 \\
$51-60$ & 17 & 28,2 & 19 & 31,7 & 36 & 30,0 \\
$61-70$ & 15 & 25,0 & 14 & 23,3 & 29 & 24,2 \\
$71-80$ & 10 & 16,7 & 09 & 15,0 & 19 & 15,8 \\
$81-90$ & 03 & 5,0 & 00 & 0 & 03 & 2,5 \\
$91-100$ & 00 & 0 & 01 & 1,7 & 01 & 0,8 \\
Total & 60 & 100,0 & 60 & 100,0 & 120 & 100,0 \\
\hline
\end{tabular}

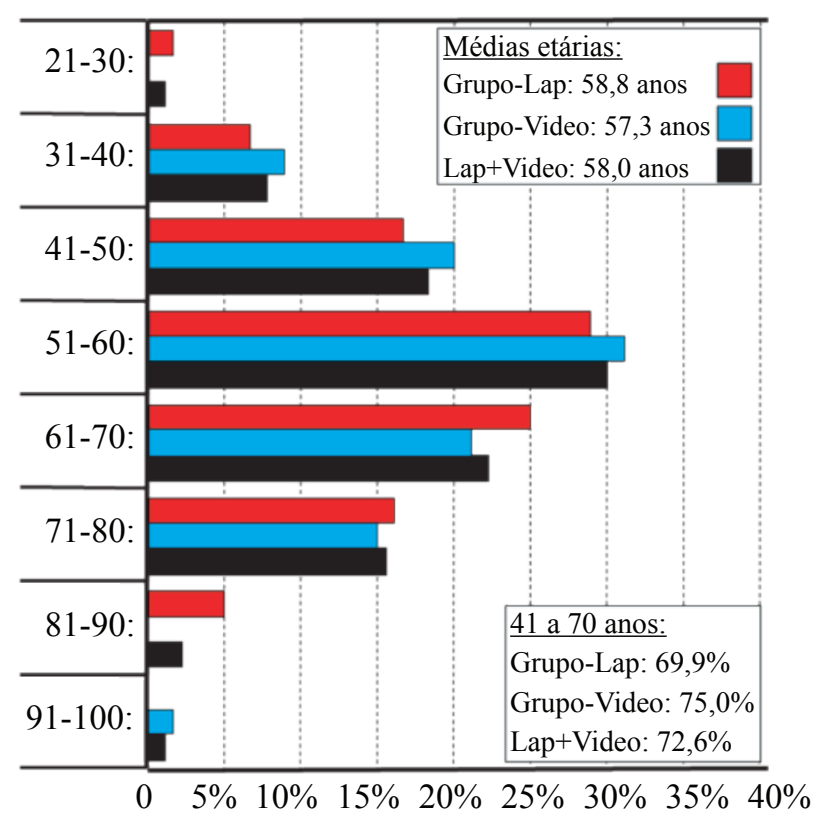

Figura 2. Distribuição, por décadas etárias, de 120 pacientes portadores de câncer colorretal submetidos a cirurgias colorretais por laparotomia (60) e videolaparoscopia (60): as semelhanças das três colunas de cada conjunto de três comprovam as semelhanças dos dados: média etária de 58,8 anos no grupo-Lap, de 57,3 anos no grupo-Video e de 58,0 anos na soma dos dois grupos; e mostram também as semelhanças das três décadas prevalentes (de 41-70 anos) para todos os grupos (grupo-Lap 69,9\%, grupoVideo $75,0 \%$ e a soma dos dois grupos $72,6 \%$ ).

$(55,0 \%)$. Somando-se as retossigmoidectomias abdominais com anastomose colorretal baixa (duplo grampeamento), o total das referidas cirurgias su- biu para 71 casos $(59,1 \%)$, concorrendo o grupoLap com 32 casos $(53,3 \%)$ e o grupo-Video com 39 casos $(65,0 \%)$. Em segundo lugar, ficou a hemicolectomia direita com anastomose íleo-transverso, com 29 casos $(24,2 \%)$, dos quais 16 no grupo-Lap $(26,6 \%)$ e 13 no grupo-Video $(21,7 \%)$ (Tabela 4 e Figura 4).

\section{Extensão das peças cirúrgicas} independentemente da cirurgia realizada

A maioria das peças cirúrgicas mediu entre 31 e $40 \mathrm{~cm}$, com média de 35,6 cm (55 casos; 45,8\%); dentro desses mesmos limites ficaram 19 peças do grupo-Lap, com média de $24,0 \mathrm{~cm}$, e 36 peças do grupo-Video, com média de $26,2 \mathrm{~cm}$. Quando se consideram todas as 120 peças cirúrgicas, a média do grupo-Lap ficou em $46,1 \mathrm{~cm}$, a do grupo-Video em $30,0 \mathrm{~cm}$ e a somatória dos dois grupos em $37,2 \mathrm{~cm}$ (Tabela 5 e Figura 5).

\section{Extensão das peças cirúrgicas dependendo da técnica cirúrgica realizada - retossigmoidectomia abdominal com anastomose colorretal}

Quando se comparam as dimensões das peças cirúrgicas oriundas de uma mesma técnica cirúrgica (71 retossigmoidectomia abdominal com anastomose colorretal), nota-se grande semelhança entre elas e as abordagens cirúrgicas por laparotomia e videolaparoscopia: média de $28,2 \mathrm{~cm}$ no grupo- 
Tabela 3. Distribuição, por localizações dos tumores no intestino grosso, de 120 pacientes portadores de câncer colorretal submetidos a cirurgias colorretais por laparotomia (30) e videolaparoscopia (60): o reto, somado ao sigmoide e ao retossigmoide, foram as principais localizações dos tumores nos dois grupos (43 no grupo-Lap, 71,7\%; 45 no grupo-Video, 75,0\%; e 88 nos dois grupos, 73,3\%) ( $p>0,05)$.

\begin{tabular}{llccccc}
\hline $\begin{array}{l}\text { Localizações } \\
\text { dos tumores }\end{array}$ & \multicolumn{2}{c}{ Grupo-Lap } & \multicolumn{2}{c}{ Grupo-Video } & \multicolumn{2}{c}{$\begin{array}{c}\text { Grupos } \\
\text { Lap+Video }\end{array}$} \\
\hline & $\mathbf{N}$ & $\mathbf{\%}$ & $\mathbf{N}$ & $\mathbf{\%}$ & $\mathbf{N}$ & $\mathbf{\%}$ \\
\hline Reto baixo (CRB) & 08 & 13,3 & 10 & 16,7 & 18 & 15,0 \\
Reto alto (CRA) & 18 & 30,0 & 10 & 16,7 & 28 & 23,3 \\
Sigmoide e retossigmoide & 17 & 28,4 & 25 & 41,6 & 42 & 35,0 \\
Descendente & 02 & 3,3 & 01 & 1,7 & 03 & 2,5 \\
Transverso & 03 & 5,0 & 02 & 3,3 & 05 & 4,2 \\
Ângulo hepático & 00 & 0 & 01 & 1,7 & 01 & 0,8 \\
Ascendente & 03 & 5,0 & 03 & 5,0 & 06 & 5,0 \\
Ceco & 09 & 15,0 & 08 & 13,3 & 17 & 14,2 \\
Total & 60 & 100,0 & 60 & 100,0 & 120 & 100,0 \\
\hline
\end{tabular}

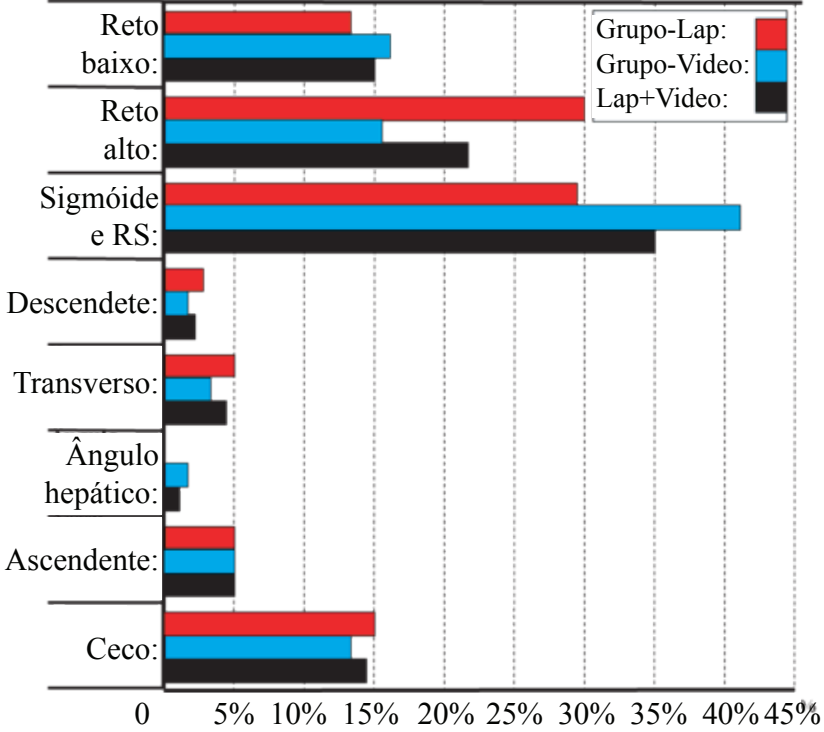

Figura 3. Distribuição, por localizações dos tumores no intestino grosso, de 120 pacientes portadores de câncer colorretal submetidos a cirurgias colorretais por laparotomia (30) e videolaparoscopia (60): os três conjuntos de três colunas comprovam a grande semelhança das localizações dos tumores colorretais no reto, sigmoide e retossigmoide (43 no grupo-Lap, 71,7\%; 45 no grupoVideo, 75,0\%; e 88 nos dois grupos, 73,3\%) ( $p>0,05)$.

Lap, de $26,6 \mathrm{~cm}$ no grupo-Video e $27,4 \mathrm{~cm}$ na somatória das duas técnicas. Nota-se também que as dimensões mais comuns foram entre 21,0 e 40,0 cm - 29 das 32 peças do grupo-Lap (90,6\%), 29 das 39 peças do grupo-Video (74,3\%), totalizando 58 das 71 peças de ambos os grupos $(81,7 \%$ ) (Tabela 6 e Figura 6).

\section{Gradação dos tumores (TNM)}

A maioria dos tumores se enquadrava no estadiamento T3N0M0, o grupo-Lap com 30 pacientes $(50,0 \%)$ e o grupo-Video com 35 pacientes $(58,4 \%)$, ficando a soma dos dois grupos com 65 casos $(54,2 \%)$. $\mathrm{O}$ segundo estadiamento mais comum (T3N1M0) coincidiu ser semelhante em ambos os grupos: 10 pacientes do grupo-Lap $(16,7 \%)$ e nove pacientes do grupo-Video $(15,0 \%)$, com a somatória dos dois grupos de 19 pacientes $(15,8 \%)$. Três pacientes do grupoVideo foram submetidos à radioterapia neo-adjuvante e após cirurgia foram estadiados como T0N0M0. (Tabela 7 e Figura 7).

\section{Contagem de linfonodos em peças cirúrgicas}

O intervalo 11 a 15 linfonodos foi o mais comum, apresentando o grupo-Lap 34 peças com média de 14,2 linfonodos (56,7\%), o grupo-Video 38 peças com média de 12,8 linfonodos $(63,3 \%)$, totalizando os dois grupos 72 peças com média de 13,4 linfonodos $(60,0 \%)$. O total de linfonodos encontrados no exame anatomopatológico das peças cirúrgicas foi 1.672, com média geral de 13,9 linfonodos por peça; no grupo-Lap, foram 810 linfonodos, com média de 13,5 linfonodos por peça, e no grupo-Video foram 862 linfonodos, com média geral de 14,3 linfonodos por peça (Tabela 8 e Figura 8).

\section{Sumário dos pacientes do grupo-Lap}

Houve mais mulheres (70 pacientes; 58,3\%) que homens (50 pacientes; $41,7 \%$ ); as décadas etá- 
Tabela 4. Distribuição, por técnicas cirúrgicas praticadas, de 120 pacientes portadores de câncer colorretal submetidos a cirurgias colorretais por laparotomia (60) e videolaparoscopia (60): a retossigmoidectomia abdominal com anastomose colorretal foi a cirurgia mais realizada no grupo-Lap (27 casos; 45,0\%) e no grupoVideo (33 casos; 55,0\%), totalizando 60 casos (50,0\%). A segunda cirurgia mais realizada foi a hemicolectomia direita com anastomose íleo-transverso, apresentando o grupo-Lap 16 casos (26,6\%) e o grupo-Video 13 casos $(21,7 \%)$, totalizando 29 casos $(24,2 \%)$.

\begin{tabular}{|c|c|c|c|c|c|c|}
\hline \multirow[t]{2}{*}{$\begin{array}{l}\text { Cirurgias } \\
\text { realizadas }\end{array}$} & \multicolumn{2}{|c|}{$\begin{array}{l}\text { Grupo } \\
\text { Lap }\end{array}$} & \multicolumn{2}{|c|}{$\begin{array}{l}\text { Grupo } \\
\text { Video }\end{array}$} & \multicolumn{2}{|c|}{$\begin{array}{c}\text { Grupos } \\
\text { Lap+Video }\end{array}$} \\
\hline & $\mathbf{N}$ & $\%$ & $\mathbf{N}$ & $\%$ & $\mathbf{N}$ & $\%$ \\
\hline Amputação abdominoperineal & 03 & 5,0 & 05 & 8,3 & 08 & $\overline{6,6}$ \\
\hline $\begin{array}{l}\text { Retossigmoidectomia com } \\
\text { ACRA* }\end{array}$ & 27 & 45,0 & 33 & 55,0 & 60 & 50,0 \\
\hline $\begin{array}{l}\text { Retossigmoidectomia com } \\
\text { ACRB** }\end{array}$ & 05 & 8,3 & 06 & 10,0 & 11 & 9,1 \\
\hline Colectomia total com AIR A $^{* * *}$ & 05 & 8,3 & 01 & 1,7 & 06 & 5,0 \\
\hline $\begin{array}{l}\text { Proctocolectomia total com } \\
\text { ileostomia }\end{array}$ & 02 & 3,4 & 00 & 0 & 02 & 1,7 \\
\hline $\begin{array}{l}\text { Hemicolectomia esquerda } \\
\text { com ATR }{ }^{\&}\end{array}$ & 02 & 3,4 & 02 & 3,3 & 04 & 3,4 \\
\hline $\begin{array}{l}\text { Hemicolectomia direita com } \\
\text { AIT }^{\& \&}\end{array}$ & 16 & 26,6 & 13 & 21,7 & 29 & 24,2 \\
\hline Total & 60 & 100,0 & 60 & 100,0 & 120 & 100,0 \\
\hline
\end{tabular}

*ACRA: anastomose colorretal alta; **ACRB: anastomose colorretal baixa; ***AIR: anastomose ileorretal; \&: ATR: anastomose transverso-retal; \&\&: AIT: anastomose íleo-transverso.

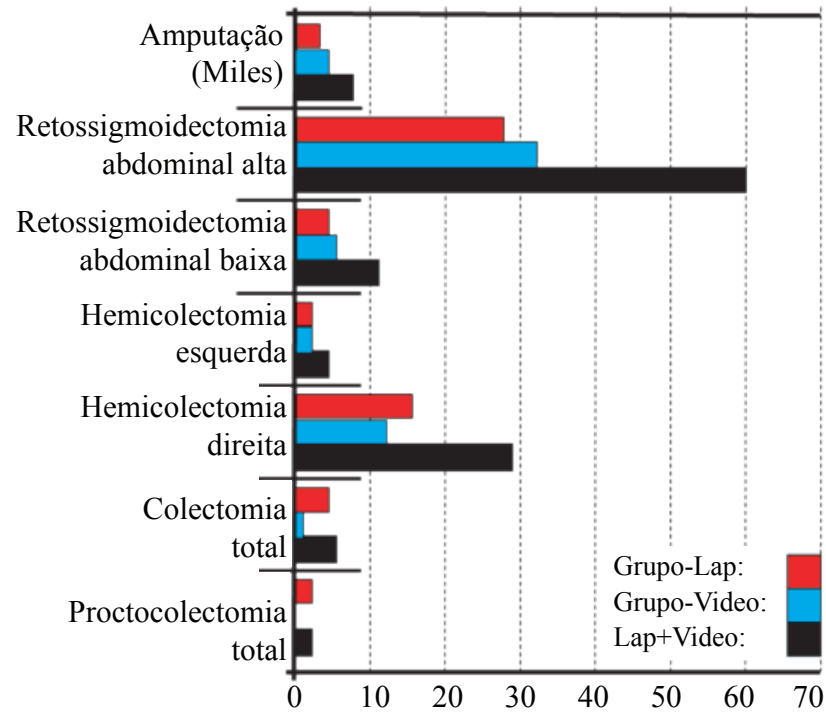

Figura 4. Distribuição, por técnicas cirúrgicas praticadas, de 120 pacientes portadores de câncer colorretal submetidos a cirurgias colorretais por laparotomia (60) e videolaparoscopia (60): o conjunto das três primeiras colunas mostram que a retossigmoidectomia abdominal com anastomose colorretal foi a cirurgia mais realizada no grupo-Lap (27 casos; 45,0\%) e no grupo-Video (33 casos; 55,0\%), totalizando 60 casos (50,0\%), seguida pelo conjunto das três colunas representando as hemicolectomias direitas com anastomose íleo-transverso. rias com maior número de pacientes foram as quinta, sexta e sétima, assim distribuídas: 10 pacientes entre 41 e 50 anos $(16,7 \%), 17$ pacientes entre 51 e 60 anos $(28,2 \%)$, e 15 pacientes entre 61 e 70 anos $(25 \%)$, totalizando 42 pacientes $(69,9 \%) ; 26$ tumores se localizavam no reto $(43,3 \%)$ e 17 no retossigmoide e sigmoide $(28,4 \%)$, totalizando $43(71,7 \%)$, seguindo o ceco com nove casos $(15,0 \%)$; a maioria das cirurgias foi representada pela retossigmoidectomia abdominal com anastomose colorretal com 32 casos $(53,3 \%)$, seguindo a hemicolectomia direita com anastomose íleo-transverso com 16 casos $(26,6 \%)$; a maioria das peças cirúrgicas mediu entre 31 e 40 cm (19 peças com média de $24,0 \mathrm{~cm}$ ); a média das extensões das peças cirúrgicas ficou em $28,2 \mathrm{~cm}$ e as dimensões mais comuns foram entre 21,0 e $40,0 \mathrm{~cm}$ (29 das 32 peças; 90,6\%); a maioria dos tumores se enquadrava no estadiamento T3N0M0 (30 pacientes; $50,0 \%$ ), seguido pelo estágio T3N1M0 com 10 pacientes (16,7\%); o intervalo 11 a 15 gânglios foi o mais comum (34 peças com média de 14,2 gânglios; $56,7 \%$ ); e o total de gânglios encontrados ao exame anatomopatológico das peças cirúrgicas foi 810 gânglios, com média de 13,5 linfonodos por peça. 
Tabela 5. Distribuição, por extensões das peças cirúrgicas ressecadas, independentemente das realizadas, de 120 pacientes portadores de câncer colorretal submetidos a cirurgias colorretais por laparotomia (60) $e$ videolaparoscopia (60): a média das extensões das peças cirúrgicas no grupo-Lap foi 46,1 cm, com 18 peças (35,2\%) com a média de 35,2 cm e 19 (31,7\%) peças com média de 24,0 cm; as médias das extensões das peças cirúrgicas do grupo-Video foi 30,0 cm, com 36 peças (60,0\%) com média de 26,2 cm e 12 peças (20,0\%) com média de 18,2 cm; a média da soma dos dois grupos foi 37,2 cm $(p<0,05)$.

\begin{tabular}{lccccccccc}
\hline $\begin{array}{l}\text { Extensão de } \\
\text { peças (cm) }\end{array}$ & \multicolumn{3}{c}{$\begin{array}{c}\text { Grupo-Lap } \\
\text { Média: 46,1 cm }\end{array}$} & \multicolumn{3}{c}{$\begin{array}{c}\text { Grupo-Video } \\
\text { Média: 30,0 cm }\end{array}$} & \multicolumn{2}{c}{$\begin{array}{c}\text { Grupos Lap+Video } \\
\text { Média: 37,2 cm }\end{array}$} \\
\hline $\begin{array}{l}\text { Todas as } \\
\text { cirurgias }\end{array}$ & $\mathbf{N}$ & Média & $\mathbf{~ \%}$ & $\mathbf{N}$ & Média & $\mathbf{\%}$ & N & Média & \% \\
\hline $15-20$ & 03 & 16,2 & 5,0 & 12 & 18,2 & 20,0 & 15 & 17,8 & 12,5 \\
$21-30$ & 19 & 24,0 & 31,7 & 36 & 26,2 & 60,0 & 55 & 25,4 & 45,8 \\
$31-40$ & 18 & 35,2 & 30,0 & 05 & 37,0 & 8,3 & 23 & 35,6 & 19,2 \\
$41-50$ & 02 & 45,0 & 3,3 & 03 & 48,1 & 5,0 & 05 & 46,9 & 4,2 \\
$51-60$ & 06 & 55,0 & 10,0 & 03 & 55,8 & 5,0 & 09 & 55,3 & 7,5 \\
$61-70$ & 05 & 67,5 & 8,3 & 00 & & 0 & 05 & 65,7 & 4,2 \\
$71-80$ & 01 & 80 & 1,7 & 00 & & 0 & 01 & 80,0 & 0,8 \\
$81-90$ & 01 & 85 & 1,7 & 00 & & 0 & 01 & 85,0 & 0,8 \\
$>100$ & 05 & 142,0 & 8,3 & 01 & 140,0 & 1,7 & 06 & 141,6 & 5,0 \\
Totais & 60 & & 100,0 & 60 & & 100,0 & 120 & & 100,0 \\
\hline
\end{tabular}

Dimensões de peças cirúrgicas (cm):

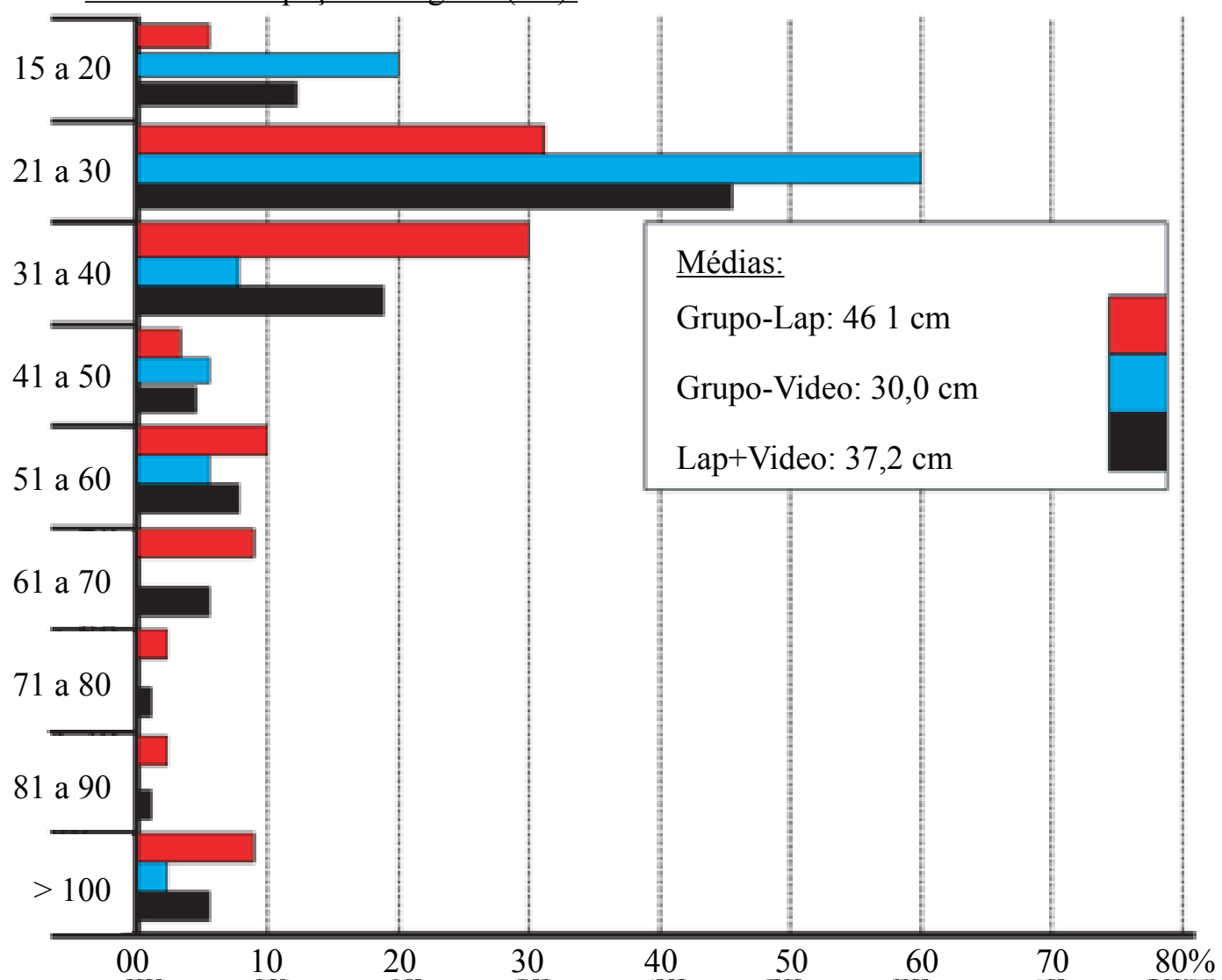

Figura 5. Distribuição, por extensões das peças cirúrgicas ressecadas, independentemente das realizadas, de 120 pacientes portadores de câncer colorretal submetidos a cirurgias colorretais por laparotomia (60) e videolaparoscopia (60): o conjunto das três colunas "21 a 30 " mostra ter sido esta a faixa mais comum de extensão das peças cirúrgicas, seguida pelo conjunto "31 a 40", com maior extensão para a segunda coluna de "21 a 30" (grupo-Lap) ( $<<0,05)$. 
Tabela 6. Distribuição, por extensões das peças cirúrgicas ressecadas, dependendo das realizadas (retossigmoidectomia abdominal), de 120 pacientes portadores de câncer colorretal submetidos a cirurgias colorretais por laparotomia (60) e videolaparoscopia (60): quando levantadas as extensões das peças cirúrgicas em relação a uma mesma cirurgia (retossigmoidectomia abdominal), verifica-se que as médias de extensões dessas são muito semelhantes - grupo-Lap (média: 28,2 cm), grupo-Video (média: 26,6 cm) e soma dos dois grupos (média: 27,4 cm) ( $p>0,05)$.

\begin{tabular}{lccccccccc}
\hline Extensão de peças & \multicolumn{3}{c}{$\begin{array}{c}\text { Grupo-Lap } \\
\text { Média=28,2 cm }\end{array}$} & \multicolumn{3}{c}{$\begin{array}{c}\text { Grupo-Video } \\
\text { Média=26,6 cm }\end{array}$} & \multicolumn{2}{c}{$\begin{array}{c}\text { Grupos Lap+Video } \\
\text { Média=27,4 cm }\end{array}$} \\
\hline $\begin{array}{l}\text { Retossigmoi- } \\
\text { dectomias }\end{array}$ & $\mathbf{N}$ & Média & $\mathbf{\%}$ & $\mathbf{N}$ & Média & $\mathbf{\%}$ & N & Média & \% \\
\hline $15-20 \mathrm{~cm}$ & 03 & 16,0 & 9,4 & 09 & 18,2 & 23,1 & 12 & 17,6 & 16,9 \\
$21-30 \mathrm{~cm}$ & 16 & 25,2 & 50,0 & 28 & 27,8 & 71,8 & 44 & 26,8 & 62,0 \\
$31-40 \mathrm{~cm}$ & 13 & 33,0 & 40,6 & 01 & 40,0 & 2,5 & 14 & 33,5 & 19,7 \\
$41-50 \mathrm{~cm}$ & 0 & & & 01 & 45 & 2,5 & 01 & 45 & 1,4 \\
Totais & 32 & 28,2 & 100,0 & 39 & 26,6 & 100,0 & 71 & 27,4 & 100,0 \\
\hline
\end{tabular}

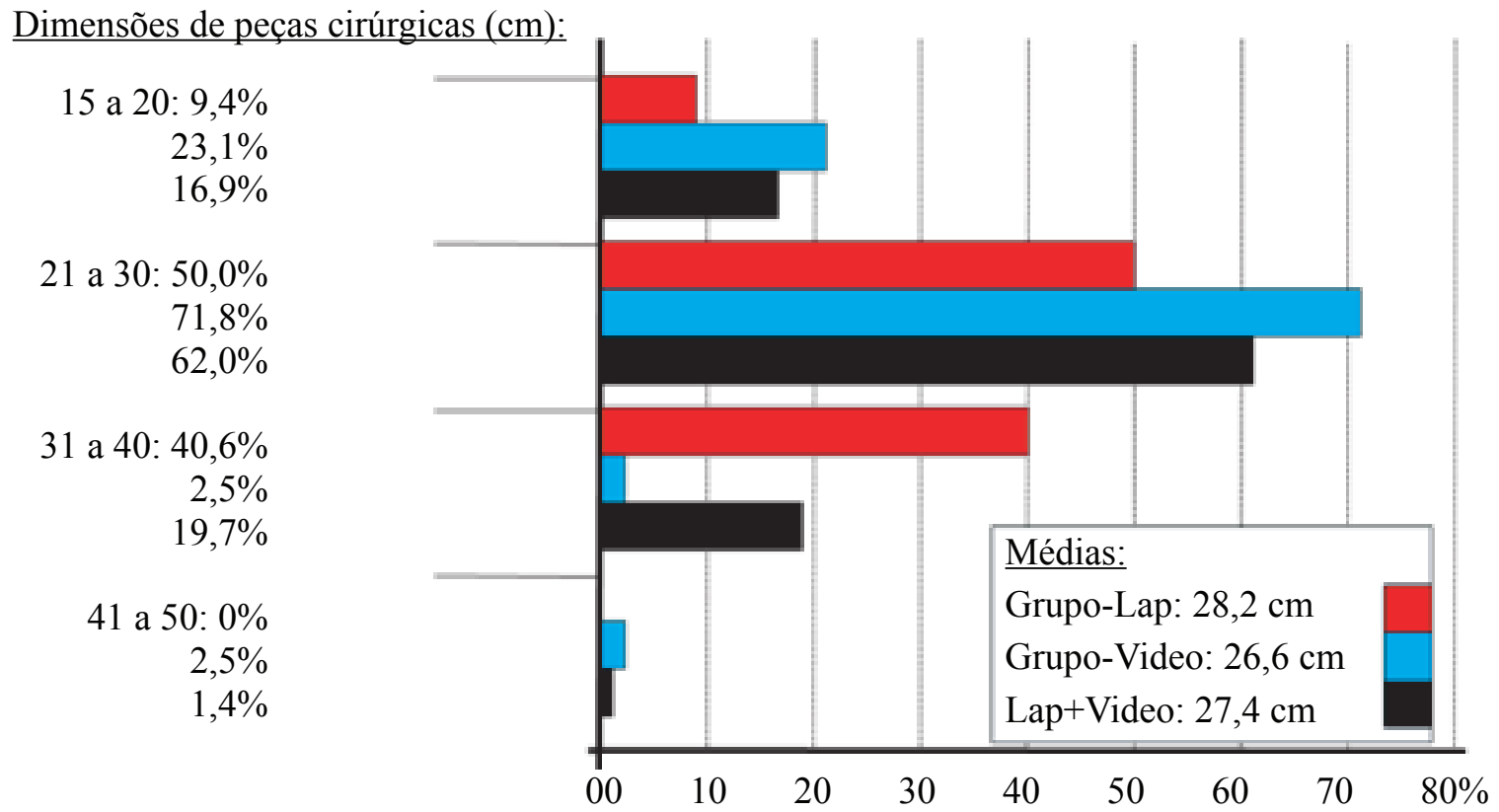

Figura 6. Distribuição, por extensões das peças cirúrgicas ressecadas, dependendo das realizadas (retossigmoidectomia abdominal), de 120 pacientes portadores de câncer colorretal submetidos a cirurgias colorretais por laparotomia (60) e videolaparoscopia (60): quando levantadas as extensões das peças cirúrgicas em relação a uma mesma cirurgia (retossigmoidectomia abdominal), verifica-se grande semelhança das colunas representativas dos dois grupos - grupo-Lap (média: 28,2 cm), grupo-Video (média: 26,6 cm) e soma dos dois grupos (média: 27,4 cm) ( $p>0,05)$.

\section{Sumário dos pacientes do grupo-Video}

Houve mais mulheres (37 pacientes; 61,7\%) que homens (23 pacientes; 38,3\%); o maior número de pacientes foi na quinta, sexta e sétima décadas, com 12 pacientes entre 41 e 50 anos $(20,0 \%), 19$ pacientes entre 51 e 60 anos (31,7\%), e 14 pacientes entre 61 e 70 anos $(23,3 \%)$, totalizando 45 pacientes $(75,0 \%)$, e a média das idades foi 57,$3 ; 20$ tumores se localizavam no reto $(33,4 \%)$ e 25 se localizavam no sigmoide e retossigmoide $(41,6 \%)$, totalizando $45(75,0 \%)$, seguido pela localização cecal com oito casos $(13,3 \%)$; a cirurgia mais realizada foi a retossigmoidectomia abdominal com anastomose colorretal com 39 casos $(65,0 \%)$, seguida pela hemicolectomia direita com anastomose íleo-transverso, com 13 casos $(21,7 \%)$; a maioria das peças cirúrgicas mediu entre 31 e $40 \mathrm{~cm}$, com 36 pe- 
Tabela 7. Distribuição, por gradação dos tumores pelo TNM, de 120 pacientes portadores de câncer colorretal submetidos a cirurgias colorretais por laparotomia (60) e videolaparoscopia (60), de maio de 2009 a maio de 2010: tanto no grupo-Lap quanto no grupo-Video, a maioria dos tumores enquadravamse no estágio T3NOMO - grupo-Lap com 30 casos $(50,0 \%)$ e grupo-Video com 35 casos $(58,4 \%)$, ficando os dois grupos com 65 casos $(54,2 \%)(p>0,05)$.

\begin{tabular}{lcccccr}
\hline TNM & \multicolumn{3}{c}{ Grupo-Lap } & \multicolumn{2}{c}{$\begin{array}{c}\text { Grupo- } \\
\text { Video }\end{array}$} & \multicolumn{2}{c}{$\begin{array}{c}\text { Grupos } \\
\text { Lap+Video }\end{array}$} \\
\hline T0N0M0 & 00 & 0 & 03 & 5,0 & 03 & 2,5 \\
T1N0M0 & 01 & 1,7 & 00 & 0 & 01 & 0,8 \\
T2N0M0 & 13 & 21,7 & 05 & 8,3 & 18 & 15,0 \\
T3N0M0 & 30 & 50,0 & 35 & 58,4 & 65 & 54,2 \\
T2N1M0 & 00 & 0 & 02 & 3,3 & 02 & 1,7 \\
T3N1M0 & 10 & 16,7 & 09 & 15,0 & 19 & 15,8 \\
T3N2M0 & 00 & 0 & 02 & 3,3 & 02 & 1,7 \\
T3N0M1 & 00 & 0 & 01 & 1,7 & 01 & 0,8 \\
T3N1M1 & 02 & 3,3 & 02 & 3,3 & 04 & 3,3 \\
T4N1M0 & 02 & 3,3 & 00 & 0 & 02 & 0 \\
T4N1M1 & 02 & 3,3 & 01 & 1,7 & 03 & 2,5 \\
Total & 60 & 100,0 & 60 & 100,0 & 120 & 100,0 \\
\hline
\end{tabular}

ças, com média de $26,2 \mathrm{~cm}$ e média global de $30,0 \mathrm{~cm}$; as dimensões das peças cirúrgicas eram oriundas de uma mesma técnica cirúrgica; a maioria dos tumores se enquadrava no estadiamento T3N0M0 (35 pacientes; $58,4 \%$ ), sendo o segundo estadiamento mais comum o T3N1M0 (nove pacientes; $15,0 \%$ ); o intervalo 11 a 15 gânglios foi o mais comum, apresentando 38 peças uma média de 12,8 gânglios (63,3\%), com número total de 862 gânglios e média geral de 14,3 gânglios por peça.

\section{DISCUSSÃO}

Apesar das preocupações iniciais sobre a utilização da técnica videolaparoscópica para a remoção de câncer de cólon, uma série de estudos foram realizados para apoiar a sua equivalência com o método convencional, e os dados apresentados por quatro estudos prospectivos randomizados controlados são convincentes $^{8-12}$, com taxas de recorrência e sobrevivência equivalentes à cirurgia laparotômica.

Os procedimentos videolaparoscópicos e seu treinamento são desafiadores, às vezes difíceis, no serviço

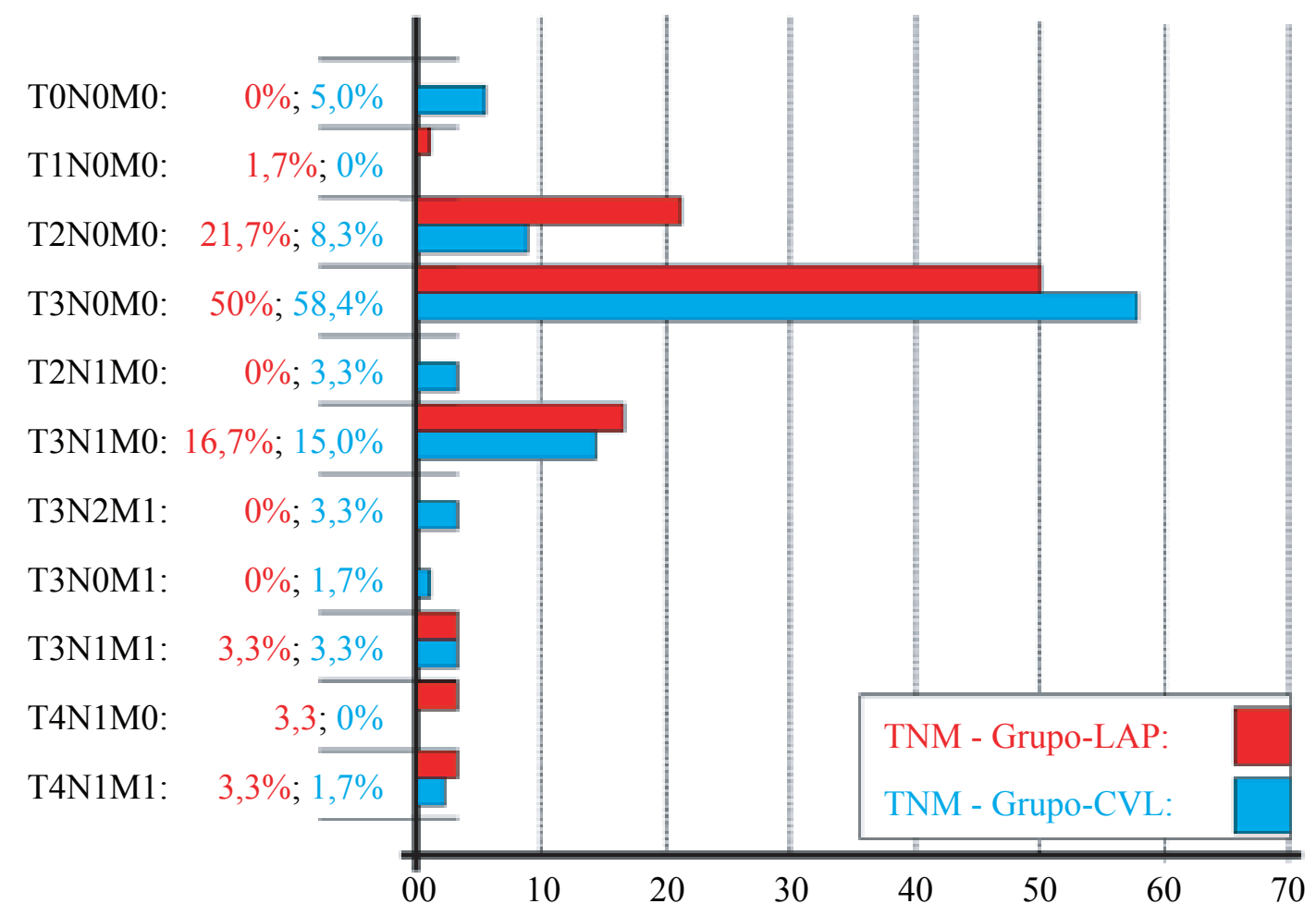

Figura 7. Distribuição, por gradação dos tumores pelo TNM, de 120 pacientes portadores de câncer colorretal submetidos a cirurgias colorretais por laparotomia (60) e videolaparoscopia (60), de maio de 2009 a maio de 2010: tanto no grupo-Lap quanto no grupo-Video, a maioria dos tumores enquadravam-se no estágio T3NOMO - grupo-Lap com 30 casos (50,0\%) e grupo-Video com 35 casos (58,4\%), ficando os dois grupos com 65 casos $(54,2 \%)(p>0,05)$. 
Tabela 8. Distribuição, por contagem do gânglios das peças cirúrgicas, de 120 pacientes portadores de câncer colorretal submetidos a cirurgias colorretais por laparotomia (60) e videolaparoscopia (60): o número total de gânglios das 120 peças cirúrgicas foi 1.672, correspondendo a uma média de 14,0 gânglios por peça cirúrgica; nas 60 peças do grupo-Lap, haviam 810 gânglios (média de 13,5 gânglios por peça), e, no grupo-Video, haviam 862 gânglios (média de 14,3 gânglios por peça) ( $p>0,05)$.

\begin{tabular}{|c|c|c|c|c|c|c|c|c|c|}
\hline \multirow[t]{2}{*}{$\begin{array}{l}\text { N de gânglios } \\
\text { nas peças }\end{array}$} & \multicolumn{3}{|c|}{$\begin{array}{c}\text { Grupo-Lap } \\
\mathrm{N}=810 ; \text { Média }=13,5\end{array}$} & \multicolumn{3}{|c|}{$\begin{array}{c}\text { Grupo-Video } \\
\text { N=862; Média }=14,3\end{array}$} & \multicolumn{3}{|c|}{$\begin{array}{c}\text { Grupos } \\
\text { Lap+Video }\end{array}$} \\
\hline & $\mathbf{N}$ & Média & $\%$ & $\mathbf{N}$ & Média & $\%$ & $\mathbf{N}$ & Média & $\%$ \\
\hline$<05$ & 02 & 4,0 & 3,3 & 00 & 0 & 0 & 02 & 4,4 & 1,7 \\
\hline $06-10$ & 15 & 8,2 & 25,0 & 12 & 8 & 20,0 & 27 & 8,1 & 22,5 \\
\hline $11-15$ & 34 & 14,2 & 56,7 & 38 & 12,8 & 63,3 & 72 & 13,4 & 60,0 \\
\hline $16-20$ & 05 & 18,2 & 8,4 & 08 & 17,8 & 13,3 & 13 & 17,9 & 10,8 \\
\hline $21-25$ & 02 & 23 & 3,3 & 01 & 23 & 1,7 & 03 & 23 & 2,5 \\
\hline $25-30$ & 02 & 30 & 3,3 & 01 & 30 & 1,7 & 03 & & 2,5 \\
\hline Total & 60 & & 100,0 & 60 & & 100,0 & 120 & & 100,0 \\
\hline
\end{tabular}

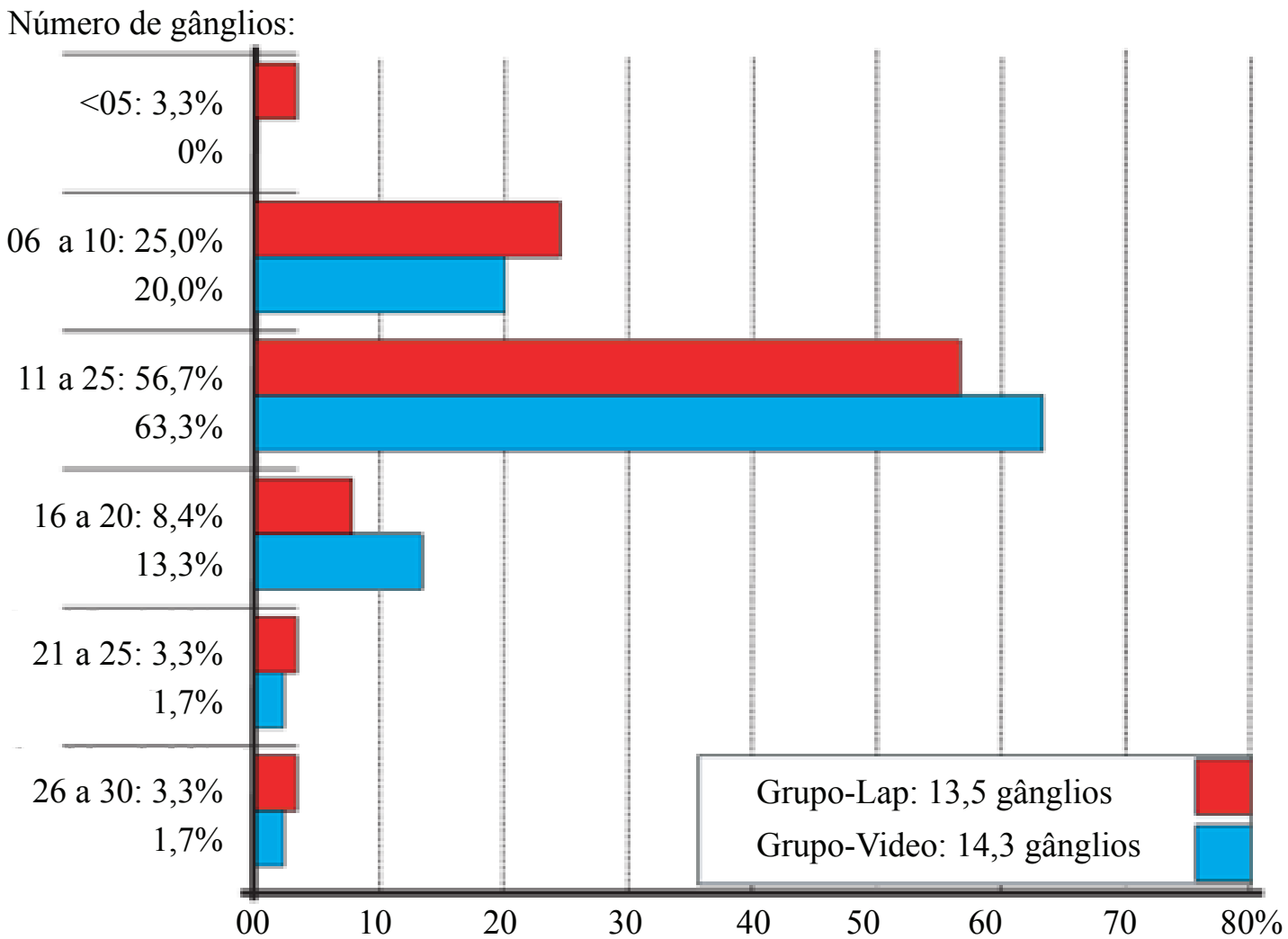

Figura 8. Distribuição, por contagem do gânglios das peças cirúrgicas, de 120 pacientes portadores de câncer colorretal submetidos a cirurgias colorretais por laparotomia (60) e videolaparoscopia (60): as duas colunas de cada conjunto mostram a grande semelhança dos números de gânglios de ambos os grupos - média de 13,5 gânglios por peça no grupo-Lap e média de 14,3 gânglios por peça no grupoVideo $(p>0,05)$.

público brasileiro. A curva de aprendizado é variável na literatura, ficando entre $40-50$ casos $^{13}$.

No presente estudo, encontramos predomínio do gênero feminino $(58,3 \%$ do total), prevalente no grupo-Video $(61,7 \%)$.
A média de idade encontrada neste estudo foi semelhante nos dois grupos, como o encontrado nas maiores séries da literatura ${ }^{12-15}$ (grupo-LAP 58,8 anos, grupo-Video 57,3 anos, com média de 58 anos), sendo prevalente a faixa etária de 41 a 70 anos $(72,6 \%$ do total $)(p<0,05)$. 
Com relação à localização do tumor, houve predomínio do reto $(38,3 \%)$, seguido pelo sigmoide e retossigmoide $(35 \%)$, resultado semelhante ao trabalho realizado por Pereira Jr et al. no Departamento de Anatomia Patológica da UFMG ${ }^{14}$, onde foram estudados 476 casos de CCR operados no Hospital das Clínicas da UFMG ${ }^{14}$. Por esse motivo, a cirurgia mais realizada foi a retossigmoidectomia $(59,1 \%)$, similar ao estudo de Kotze et al ${ }^{15}$.

Como o trabalho realizado foi uma análise comparativa da cirurgia convencional com a laparoscopia para câncer colorretal, o tamanho das peças cirúrgicas variou de $15 \mathrm{~cm}$ a mais de $100 \mathrm{~cm}$ (média de $37,2 \mathrm{~cm}$ ). Para diminuir a diferença e permitir comparação entre as técnicas, analisamos o tamanho das peças das retossigmoidectomias, maioria dos procedimentos $(59,1 \%)$. Pela retossigmoidectomia, o tamanho das peças ficou entre 21 e $40 \mathrm{~cm}$, com média de $28,2 \mathrm{~cm}$, não havendo diferença estatística entre as técnicas e mostrando-se semelhante a outros estudos ${ }^{16}$.

Quanto ao estadiamento TNM, o mais encontrado nos dois grupos foi o T3N0M0 $(54,2 \%)$, prevalência no grupo-Lap $(58,4 \%)$, seguido pelo T3N1M0 $(15,8 \%)$. Pacientes com estádio maior que E2, totalizaram $84,4 \%$ do nosso estudo, o que é muito maior que o estudo realizado no Japão ${ }^{1}$, no qual encontraram $40 \%$ dos pacientes com esse estádio, porém mais próximo ao estudo de Pereira $\mathrm{Jr}$ et $\mathrm{al}^{14}$. $\mathrm{O}$ resultado similar dos estudos brasileiros e diferentes do japonês pode ser justificado pela dificuldade de acesso dos brasileiros ao serviço público de saúde do país, ocasionando demora no diagnóstico e tratamento.

$\mathrm{O}$ número médio de linfonodos encontrados nos dois grupos foi de 13,9, não havendo diferença estatística das médias entre eles (13,5 grupo-Lap e 14,3 no grupo-Vídeo, $p>0,05)$. No presente estudo, a média de linfonodos ficou acima do que é preconizado pelas principais diretrizes oncológicas, 12 linfonodos por peça cirúrgica ${ }^{17-19}$, e maior que a maioria dos estudos comparativos já realizados ${ }^{8-11,13}$.

\section{CONCLUSÃO}

Não houve ou houve diferenças sem significado estatístico entre os dois grupos em: gênero, idade, incidência de tumores retais altos e baixos, número de retossigmoidectomias, extensões de peças cirúrgicas, gradação TNM dos tumores e média de gânglios linfáticos por peça cirúrgica.

O trabalho em questão sugere que ambas as técnicas (laparotomia e laparoscopia) são igualmente eficazes no tratamento do câncer colorretal já que não encontramos diferenças significativas (critérios oncológicos estudados) entre os dois grupos.

ABSTRACT: The objective was to undertake a careful review of a consecutive series of 120 patients with colorectal cancer approached by laparoscopic surgery (Video-group, 60 patients) and by laparotomy (Lap-group, 60 patients), during 12 months (May 2009 to May 2010). The average age was 58 years and 58.8 years in group-Lap and 57.3 years in Video-group, the majority being females in both groups (Lap-group - 55.0\% and Video-group - 61.7\%, general mean 58.3 years). All patients in both groups underwent colonoscopy and biopsy with histopathologic diagnosis of adenocarcinoma. As far as distribution of tumors in the large intestine in Lap-group, 43 tumors were located in the rectum, rectosigmoid and sigmoid colon (71.7\%) versus 45 in Video-group (75.0\%), but with differences between low rectum (Lap-group 13,3\%, Video-group 16.7\%) and upper rectum (Lap-group 30.0\%, Video-group 16.7\%), sigmoid and rectosigmoid (Lap-group 28.4\%, Video-group 41.6\%). The most performed surgery was abdominal rectosigmoidectomy (Lap-group 27 cases, 45.0\%; and Video-group 33 cases, 55,0\%), followed by right hemicolectomy (Lap-group 16 cases, 26,6\%; and Video-group 13 cases, $21.7 \%$ ). The extensions of the surgical specimens were greater in Lap-group (mean $46.1 \mathrm{~cm}$ vs. $30.0 \mathrm{~cm}$ in Video-group), but due to higher number of surgeries that resulted in longer specimens. When comparing same surgical techniques, the difference does not persist as in cases of retosigmoidectomy (Lap-group with 32 cases, mean $28.2 \mathrm{~cm}$; and Video-group with 39 cases, mean $26.6 \mathrm{~cm}$ ). With regard to TNM staging T3N0M0, tumors was the most common in Lap-group with 30 cases $(\mathbf{5 0 . 0 \% )}$ and Video-group with 35 cases $(\mathbf{5 8 . 4 \% )}$ ). Regarding the lymph nodes count in surgical specimens, no difference was noted: total of 810 lymph nodes in specimens of Lap-group with a mean of 13.5 nodes per specimen, and total of 862 lymph nodes in Video-group with an average of 14.3 nodes per specimen. No difference was noted in relation to the count of lymph nodes in surgical specimens: the number of nodes was most commonly between 11 and 15 per specimen: 34 cases in Lap-group (56.7\%) and 38 in Video-group (63.3\%). Thus, no difference was noted between the two groups (Lap-group and Video-group) as far as oncologic and surgical criteria are concerned.

Keywords: rectal cancer; laparoscopy; colorectal surgery. 


\section{REFERÊNCIAS}

\section{Pós-graduação sensu lato pelo Grupo de Coloproctologia de Belo Horizonte} José Roberto Monteiro Constantino e Cols.

1. Inomata M, Yasuda K, Shiraishi N, Kitano S. Clinical evidences of laparoscopic versus open surgery for colorectal cancer. Jpn J Clin Oncol 2009;39(8):471-7.

2. Champagne BJ, Delaney CP. Laparoscopic approaches to rectal cancer. Clin Colon Rectal Surg 2007;20(3):237-48.

3. Kahnamoui K, Cadeddu M, Farrokhyar F, Anvari M. Laparoscopic surgery for colon cancer: a systematic review. Can J Surg 2007;50(1):48-57.

4. Regadas FSP, Regadas SMM, Rodrigues LV, Lima D, Silva FR, Regadas Filho FSP. Cirurgia colorretal laparoscópica. Experiência com 401 casos. Rev bras de videocir 2005;3(4):191-5.

5. Ministério da Saúde (BR), Instituto Nacional de Câncer, Secretaria de Atenção à Saúde, Coordenação de Prevalência e Vigilância. Estimativa 2005: incidência do câncer no Brasil. Rio de Janeiro (Brasil): Ministério da Saúde (BR), INCA; 2004.

6. Tonon LM, Secoli SR, Caponero R. Câncer colorretal: uma revisão da abordagem terapêutica com bevacizumabe. Revista Brasileira de Cancerologia 2007;53(2):173-82.

7. Sistema Único de Saúde. Datasus. Brasil: C. 10 n. de mortalidade específica por neoplasia maligna - cólon, reto e ânus, por sexo, segundo faixa etária, em 2002. Disponível em: http://tabnet.datasus.gov.br/cgi/tabcgi.exe?idb2004/c10.def.

8. Lacy AM, García-Valdecasas JC, Delgado S, Castells A, Taurá P, Piqué JM, et al. Laparoscopy-assisted colectomy versus open colectomy for treatment of non-metastatic colon cancer: a randomised trial. Lancet 2002; 359(9325):2224-9.

9. COST Study. A comparison of laparoscopically assisted and open colectomy for colon cancer. N Engl J Med 2004;350(20):2050-9.

10. Guillou PJ, Quirke P, Thorpe H, Walker J, Jayne DG, Smith AM, et al. Short-term endpoints of conventional versus laparoscopic-assisted surgery in patients with colorectal cancer (MRC CLASICC trial): multicentre, randomised controlled trial. Lancet 2005;365(9472):1718-26.

11. Veldkamp R, Kuhry E, Hop WC, Jeekel J, Kazemier G, Bonjer HJ, et al. Laparoscopic surgery versus open surgery for colon cancer: short-term outcomes of a randomised trial. Lancet Oncol 2005;6(7):477-84.

12. Boller AM, Nelson H. Colon and rectal cancer: laparoscopic or open? Clin Cancer Res 2007;13:6894-6.

13. Neiva AM, Lacerda Filho A, Cabral MMDA, Luz MMP, Fonseca LM, Hanan B, et al. Análise de 33 peças cirúrgicas de colectomias laparoscópicas para câncer durante a curva de aprendizado inicial: margens oncológicas e número de linfonodos não diferem de colectomias abertas. Rev bras Coloproct 2010; 30(1):07-13

14. Pereira $\mathrm{T}$ Jr, Alves AJC, Nogueira AMMF. Câncer colorretal: análise anatomopatológica de 476 colectomias consecutivas em Belo Horizonte (MG). J Bras Patol Med Lab 2005;41(3):175-84.

15. Kotze PG, Freitas CD, Froehner Júnior I, Steckert JS, Ishie E, Steckert Filho A, et al. Análise do número de linfonodos em espécimes de ressecções colorretais por neoplasia entre a cirurgia aberta e videolaparoscópica. Rev bras Coloproct 2010;30(2):119-27.

16. Melani AGF, Campos FGCM. Ressecção laparoscópica pósterapia neo-adjuvante no tratamento do câncer no reto médio e baixo. Rev bras Coloproctol 2006;26(1):89-96

17. Engstrom PF, Arnoletti JP, Benson AB, Chen YJ, Choti MA, Cooper HS, et al. NCCN Clinical Practice Guidelines in Oncology: colon cancer. J Natl Compr Canc Netw 2009; 7(8):778-831.

18. Engstrom PF, Arnoletti JP, Benson AB, Chen YJ, Choti MA, Cooper HS, et al. NCCN Clinical Practice Guidelines in Oncology: rectal cancer. J Natl Compr Canc Netw 2009;7(8):838-81.

19. Nelson H, Petrelli N, Carlin A, Couture J, Fleshman J, Guillem J, et al. Guidelines 2000 for colon and rectal cancer surgery. J Natl Cancer Inst 2001;93(8):583-96.

Endereço para correspondência:

Geraldo Magela Gomes da Cruz

Rua Rio de Janeiro, 2017/1401

CEP: 30160-042 - Belo Horizonte (MG), Brasil

E-mail: magelacruz@terra.com.br 